\title{
MUSCULOSKELETAL DISORDERS: PRIMARY AND SECONDARY INTERVENTIONS
}

\author{
Robert J. Gatchel, Ph.D., ABPP \\ Professor, Departments of Psychiatry, Anesthesiology \& Pain Management, \\ and Rehabilitation Counseling \\ The University of Texas Southwestern Medical Center at Dallas \\ 5323 Harry Hines Blvd. \\ Dallas, TX 75390-9044 \\ 214-648-5277 \\ 214-648-5297 (FAX) \\ robert.gatchel@utsouthwestern.edu
}

This research partially funded by grants No. 5K02 MH01107, 4R01 MH46452, and 5R01 DE10713 from the National Institutes of Health 
In the United States alone, the annual cost associated with the diagnosis and care of musculoskeletal trauma amounts to tens of billions of dollars (cf. Mayer, Gatchel, \& Polatin, 2000). Moreover, these costs are continuing to increase at an alarming rate. In fact, in the United States today, occupational musculoskeletal disorders are the leading causes of work disability. Changes in health care policy and demand for improved allocation of health care resources by the Federal government have also recently placed greater pressure on health care professionals to provide the most cost-effective treatment for these disorders, as well as to validate treatment effectiveness. Indeed, treatment-outcome monitoring has assumed new importance in medicine. It is particularly essential in musculoskeletal care, which is currently targeted for attention by health care planners because of its high cost and perceived traditional inefficient care. With these facts in mind, the purpose of the present article is to review the status of current primary and secondary interventions for musculoskeletal disorders. Before doing so, a brief discussion of the biopsychosocial model of pain and disability, which is currently the most heuristic approach to intervention, will be provided.

\section{THE BIOPSYCHOSOCIAL MODEL OF MUSCULOSKELETAL PAIN AND DISABILITY}

Today, the biopsychosocial approach to pain and disability is widely accepted, and has replaced the outdated biomedical reductionistic approach (Turk \& Gatchel, 2002; Turk \& Monarch, 2002). The biopsychosocial approach views pain and disability as a complex and dynamic interaction among physiologic, psychologic and social factors, which perpetuates and may worsen the clinical presentation. In stark contrast, the traditional biomedical approach assumes that there is a specific physical cause of the symptoms, and attempts are made to eradicate the cause by rectifying the physical pathology, or by cutting or blocking the pain 
pathways pharmacologically or surgically. The biomedical approach traditionally has promised a cure or, barring that, elimination of a significant amount of pain. Currently, though, there are no definitive cures for the most prevalent chronic pain syndromes such as back pain, osteoarthritis or peripheral neuropathies. Rehabilitation rather than cure are, at this point, the most appropriate therapeutic options. The outdated notion of holding out the promise for an elusive cure, followed by failure and disappointment, adversely affects musculoskeletal pain sufferers by holding out the promise of cure where none currently exists, and thereby drives up health care costs.

The range of psychologic, social and economic factors can interact with physical pathology to modulate a patient's report of symptoms and subsequent disability. This biopsychosocial model, unlike reductionistic biomedical perspectives, provides an account of the likelihood that patients' lives are greatly impacted upon by the persistent pain, thus contributing to the maintenance of pain and the associated disability. It follows from the biopsychosocial perspective that appropriate treatment requires a comprehensive approach designed to address all factors that cause, moderate, and maintain pain and disability.

\section{Disease versus Illness}

With this above biopsychosocial approach in mind, it is also important to make a distinction between disease and $\underline{\text { illness. }}$. Turk and Monarch (2002) have defined disease as an objective, biological event involving the disruption of specific body structures or organ systems due to anatomical, pathological or physiological changes. In contrast, illness is a subjective experience or self-attribution that a disease is present, creating physical discomfort, behavioral limitations, and psychosocial distress. This distinction between disease and illness is analogous to the distinction between nociception and pain. Nociception is the stimulation of nerves that 
convey information about tissue damage to the brain. In contrast, pain is a more objective perception that is the result of the transduction, transmission and modulation of sensory input. It may be filtered through an individual's genetic composition, prior learning history, current physiological status, and sociocultural influences. The biopsychosocial model focuses on illness, especially the interrelationships among biological changes, psychological status, and the sociocultural context that all need to be considered. This helps to explain the diversity of pain or illness expression, including its severity, duration, psychosocial consequences, and treatment outcomes. Any model or treatment approach that focuses on only one of these core sets of factors to the exclusion of the others will, inevitably, be incomplete and therefore inadequate. Curative versus Management Approaches

Musculoskeletal pain, especially when it becomes chronic in nature, often cannot be "cured" but only managed in a comprehensive, interdisciplinary treatment program. An example of such a program is functional restoration, first introduced by Mayer and Gatchel $(1988 ; 1987)$. This is a biopsychosocial interdisciplinary approach that focuses on addressing the interrelationships described above. As such, it demands coordinated care for patients with complex needs and requirements — psychosocial, behavioral, as well as physical. Such interventions are performance-based, goal-directed, and outcome-driven. Common features of all such programs include physical therapy, medication management, education about how the body functions, psychological treatments (e.g., coping skills, learning, problem solving, communication skills training), vocational assessment, and therapies aimed at improving function and the likelihood of return-to-work. The treatment team must build an alliance with patients to instill a willingness to accept the need for self-management. Function drives the treatment process, and not self-reported pain. As such, the basic philosophy of "management of 
pain" rather than "curing of pain" must be emphasized consistently to patients throughout the intervention process.

\section{REVIEW OF PRIMARY, SECONDARY AND TERTIARY CARE}

At the outset, it should be noted that the terms primary, secondary and tertiary may be used to refer both to treatment and prevention, but with slight differences in meaning. Primary care consists mainly of passive modalities directed towards pain relief, and is most appropriately administered to acute cases during the stages of early soft-tissue healing. Primary prevention represents interventions with the uninjured worker population and the workplace to avoid injury (and thus the subsequent need for primary care).

Secondary or "reactivation" care (such as "work hardening") represents early nonspecific exercise and education programs for the $20-30 \%$ of patients requiring a transition from acute care to return-to-work. It is therapy-driven, and is customarily provided within the first six months after injury, before chronic disability habituation supervenes. Alternatively, it is applied postoperatively as a limited rehabilitation approach. Secondary prevention refers to interventions with the freshly injured patient to avoid chronic disability habituation by efforts to return the patient to productivity as soon as possible. Both primary and secondary care may be involved in this process, along with workplace intervention and job-modification programs.

Finally, tertiary care (such as functional restoration or industrial pain management) is physician-directed, interdisciplinary, individualized and intensive treatment designed for the small fraction of patients whose biomechanical dysfunction, physical deconditioning and psychosocial stressors have led to chronic, entrenched disability. Tertiary prevention attempts avoid high costs associated with the permanent loss of productivity of the small fraction of disabled workers who ultimately become the ongoing disabled workers. Tertiary (or 
occasionally secondary) care, as well as interventions involving modified work or vocational rehabilitation, are common. A more detailed delineation of the intervention differences have been provided by Mayer et al. (1995).

In this present article, major attention will be focused on primary and secondary intervention. The basic differences between these two types of interventions are delineated below.

- Primary Care involves symptom control when acute pain predominates (usually 0-10 weeks after injury occurrence).

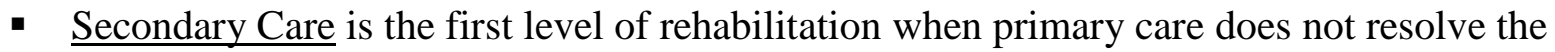
problem. The first stage of secondary care (also called secondary rehabilitation) involves reactivation during the transition from primary care to return-to-work. It is designed to facilitate return to productivity before progressive deconditioning and psychosocial barriers supervene. The rationale for secondary care is that early recognition and management of risk factors for developing disabilities can prevent chronic or permanent disability.

In the area of low back pain, there are now some clinical guidelines that have been published which, generally, can be used with other types of musculoskeletal disorders. For example, McGuirk et al. (2001) have demonstrated that the use of such guidelines achieves clinical and statistical improvement, with fewer patients requiring continuing care and remaining in pain. These guidelines emphasize the following:

- Dealing with patients' fears and misconceptions about low back pain

- Providing a confident explanation for the reason for the pain, as well as a prognosis 
- Empowering the patient to resume/restore normal activities of daily living through simple prescribed exercises and graded activity. This should be supplemented, when necessary, by simple adjunctive approaches such as analgesics and manual therapy for symptomatic relief.

More discussion of such an approach will be provided later in this article.

\section{REVIEW OF "RED, YELLOW, BLUE AND BLACK FLAGS"}

Over the last decade, there has been a growing interest in developing a systematic method of assessing acute low back pain patients who have potentially significant risk factors for developing chronic problems. Presently, the New Zealand Acute Low Back Pain Guide (ACC and the National Health Committee, 1997) is viewed as providing the most current and comprehensive information, covering both physiological (red flags) and psychosocial (yellow flags) risk factors associated with the progression from acute to chronic low back pain disability. Since that time there have been a number of other risk factor categories delineated (the blue and black flags). Table 1 presents a summary of these risk factor or "flag" categories.

\section{INSERT TABLE 1 ABOUT HERE}

\section{$\underline{\text { Red Flags }}$}

Using the guidelines, acute low back pain patients undergo an initial assessment during their first health care visit in order to determine if red flags, or potentially dangerous physiological conditions, are present. Initially, a thorough medical history focusing particularly on the patient's current symptoms and limitations, duration of symptoms, and history of previous episodes is conducted. Based on this medical history, a physical examination should be 
conducted which may include a general observation of the patient, a regional back examination, neurological screening, and a test for sciatic nerve root tension. The information gathered from the medical history and physical examination is then integrated and the patient is placed into one of three categories: simple backache; nerve root pain; or possible serious spinal pathology. The category "simple backache" refers to mechanical back pain that is musculoskeletal in nature.

"Nerve root pain" is more serious and involves unilateral leg pain and numbness, which is typically of greater intensity than the patient's low back pain. Often, such pain is precipitated by a disc prolapse, spinal stenosis, or surgical scarring. "Possible serious spinal pathology" is potentially the most threatening category, which may reflect conditions such as tumors, infections, inflammatory diseases, structural deformities of the back or widespread neurological disorders. This type of pain is often constant, progressive, does not vary in intensity depending upon one's physical activity.

In addition to gathering the above medical history/physical examination information, the primary care physician should also be investigating the presence of "red flags" during the patient's initial assessment. The presence of any red flag should be taken seriously, as it signals the potential need for the patient to undergo additional testing, or even urgent surgical evaluation. There are a number of potential red flags that must be kept in mind when examining acute low back pain patients, as delineated below.

- Age; if the patient is either less than 20 years old or greater than 55 years old

- A violent trauma, such as a fall from a height

- Constant, progressive, non-mechanical pain

- The presence of thoracic pain

- A past medical history of carcinoma 
- The use of steroids or drug abuse

- Being HIV positive and/or using intravenous drugs

- Being in poor health or physically unwell

- Weight loss

- Persistent severe restriction of lumbar flexion

- Widespread neurological symptoms

- Structural deformity

- Fever

- Severe, unremitting night time pain

- Pain that gets worse when the patient is lying down

It should be kept in mind that individual red flags by themselves do not necessarily facilitate an accurate diagnosis. Instead, the presence of red flags may be useful in determining if additional investigation should be conducted, as well as directing the primary care physician to specific types of assessment that are indicated. If a patient possesses one or more of the above red flags, he or she should be referred to a specialist for further testing. If a patient presents with more severe red flags, such as sphincter disturbance, gait disturbance, etc., a referral should be made and further study should begin immediately.

Many patients may be totally free of red flags. If that is the case, there is no need for these patients to be subjected to additional, costly diagnostic tests. Most often, they can be successfully treated by an early intervention strategy that provides symptomatic relief from pain. There are a number of important components that need to be included in this type of primary intervention (see Table 2). 


\section{INSERT TABLE 2 ABOUT HERE}

If acute low back pain patients who participate in the primary intervention approach presented in Table 2 do not experience significant decreases in pain, and have consequently not yet returned to work or to their normal activities of daily living within 4-6 weeks, then it is usually recommended that they undergo additional testing to determine if there are any potential pathophysiological underpinnings related to their low back pain. If all of these investigations are subsequently found to be normal, but the patient's symptoms still persist after six weeks, they then should be referred to an interdisciplinary treatment program dedicated to the prevention of the development of chronicity (i.e., secondary or tertiary care).

\section{Yellow Flags}

Using the biopsychosocial approach, there has been growing research aimed at identifying salient psychosocial risk factors involved in the development of chronic pain disability. Much of this research has been conducted with low back pain patients, because this is one of the most prevalent forms of musculoskeletal pain disability. Because most back pain problems are non specific, with no well delineated underlying pathophysiology or anatomical deficits, there was a need to develop an effective way of assessing psychosocial factors that contribute to disability. Initially lacking any type of standardization, the National Health Committee and Accident Rehabilitation Compensation Insurance Corporation conducted a collaborative project in New Zealand (Kendall, Linton, \& Main, 1997). The major goal of this project was to develop some evidence-based guidelines that primary care physicians might use to assess acute low back pain patients in a more effective manner. In this project, acute low back pain was defined as pain lasting less than three months in duration. As part of this project, a 
review of previously conducted research indicated that the typical treatments for this condition (namely physiological and/or pharmacological interventions) provided during the acute phase of low back pain were largely ineffective. As a result, special attention was paid to psychosocial risk factors or "yellow flags." Because such flags were deemed particularly difficult to evaluate, this project provided health care professionals with a more systematic approach to identification and quantification of such psychosocial risk factors that were believed to be intimately tied to long term disability. Moreover, a number of suggestions regarding early biobehavioral management techniques believed to prevent chronic low back pain were also made.

Yellow flags are psychosocial barriers that may impede one's ability to improve and/or recover from acute pain episodes. It should be noted that the presence of yellow flags is in no way indicative of malingering, and should be conceptualized as one of numerous variables that interact and ultimately impede the healing and recovery process. In order to facilitate a relatively “easy-to-use" method of assessment, an acronym was developed (ABCDEFW), with each letter standing for one of the seven main types of yellow flags. These are reviewed below.

- "A" stands for attitudes and beliefs about pain, and is useful in determining how a patient understands and conceptualizes his or her low back pain. Patients who view their pain to be harmful or disabling frequently develop a type of fear-avoidance behavior in which they minimize physical movement for fear they will re-injure or hurt themselves further. This type of guarded movement, if maintained for any period of time, will often result in muscle atrophy and physical deconditioning, which will ultimately lead to increased pain perception when they begin to become more mobile. Patients are also at risk for developing chronicity if they display the following: adopt a passive attitude toward their rehabilitation process; believe that their pain is uncontrollable; misinterpret their bodily 
symptoms and resort to catastrophizing; expect to experience increased pain levels when they are active; and believe that all of their pain must be relieved before they are willing to return to work.

- "B" refers to the patient's behaviors. More specifically, patients who rely heavily on rest of "downtime" may be at increased risk for developing chronic low back pain. Moreover, patients are considered to be "at risk" if they suddenly reduce their activity level and significantly withdraw from activities of daily living. This type of withdrawal may be especially problematic for acute low back pain patients because they are likely to become much more sedentary, socially isolated, and ultimately remove themselves from situations in which they may receive the social support of others. Some of the other risk factors related to behavior include: the avoidance of normal activity and increased time away from productive activity; reporting extremely high pain levels (such as a 15 on a 0 10 scale); an excessive reliance on aids or appliances; reduction of sleep quality following the onset of the acute episode; a significant increased use of alcohol or other substances following the acute onset; and smoking.

- "C" refers to compensation issues frequently associated with workers' compensation and disability payments. Patients who lack the financial incentive to return to work appear to be at risk for long term disability. Because many individuals with acute low back pain are receiving a substantial disability/workers' compensation payment, they may not entirely be motivated to return to work in a timely manner. This will become problematic because the longer an individual is out of work, the less likely he/she will gain meaningful employment within the near future. It has also been found that individuals appear to be at risk if: they experience a delay in income support, such as disability or 
workers' compensation; have a history of filing claims for other pain problems or injuries; have a tendency to take off from work for extended periods of time due to injuries or pain problems; and have had prior experience working with an ineffective and/or punitive case manager.

- "D" refers to diagnosis and treatment. It has been found that patients may be at greater risk if their physician is not careful to present their diagnosis in a gentle and reassuring manner. If this is not done appropriately, acute low back pain patients may not fully understand their diagnosis, and are more likely to become extremely fearful about their prognosis and the future. In addition, confusion and anxiety may be generated if the patient receives competing/incompatible diagnoses, or disparate explanations as to how physicians believe their low back pain began. In fact, physicians themselves may unwittingly harm their patients by encouraging them to take a leave of absence from work or remain sedentary until the pain subsides. In addition, other factors associated with the risk of chronicity development are the following: when patients are provided with treatment that only requires passive engagement on their part; they expect a "technofix," and request to be treated as if their "body were a machine"; were not satisfied with the previous treatment they received for their low back pain; or have a high frequency of health care visits within the past year for issues unrelated to the current acute low back pain episode.

- "E" refers to emotions that are believed to pay an important role in the progression of acute to chronic low back pain. Patients who feel hopeless, or as though they have nothing to offer others as the result of their pain, are usually at increased risk for the development of chronicity. Moreover, patients who have an unusually heightened 
awareness of their own bodily sensations also are at risk because they usually spend an inordinate amount of time attending to every ache and pain they experience, thereby constantly focusing on their low back pain. Because such individuals find it difficult to take their mind off their pain, this usually elevates their stress level and prevents them from focusing their energy on more productive or pleasurable activities. Furthermore, individuals are at increased risk when they are experiencing depression, anhedonia, irritability, social anxiety or disinterest in social activity, and/or high levels of stress and a perception of a lack of control.

- "F" refers to familial factors that are particularly important because they provide a glimpse of the patient's social support structure. Families/significant others who are overly solicitous and take over the patient's duties or chores are actually doing the patient more harm than good. Because all of the patient's needs are being met with only minimal effort, the patient has little or no motivation to become more mobile and to resume everyday duties. Of course, it should be noted that this is not always a deliberate act on the part of the patient, but often occurs unknowingly. In contrast, families who respond to patients in a punitive manner (either by ignoring them altogether or expressing their frustration) are also detrimental, and are believed to contribute to the development of chronicity. Finally, families that do not encourage the patient to return to work, and who may actually prohibit them from discussing their problems or concerns, also likely act to engender the development of chronicity.

- "W" refers to risk factors related to work. Individuals with a history of manual work (such as farmers, fishermen, construction workers, truck drivers, nurses, daily laborers) have a greater probability of progressing from acute to chronic low back pain. Moreover, 
jobs that involve significant biomechanical demands (such as heavy lifting, extended periods of sitting/standing, driving, vibration, maintenance of sustained postures, and inflexible work schedules that prevent appropriate breaks) produce a greater risk for development of chronicity. In addition, individuals are at greater risk if they think their work is harmful and dangerous. As a result, they may believe that if they engage in such jobs, they will physically damage themselves and experience heightened levels of pain. Finally, some additional work-related risk factors include the following: past job dissatisfaction; patterns of frequent job change; a current work environment that is unsupportive; a low educational level and low socioeconomic status; jobs that involve shift work; little, if any, availability of selected duties and graduated return-to-work pathways (such as light duty); and a workplace management system that is unsatisfactory where individuals are discouraged from filing injury reports.

Even though the initial assessment of acute patients focuses primarily on red flags, if yellow flags are concurrently present, they should be noted at that time. Indeed, if a patient is not recovering as expected, and has not regained usual activity after four weeks, he/she should be formally re-evaluated six weeks after the initial assessment, with a greater emphasis placed on identifying yellow flags that may serve as major barriers to recovery. A 24-item self-report screening questionnaire that provides primary care physicians with a general summary of the psychosocial risk factors has been developed-The Acute Low Back Pain Screening Questionnaire. Kendall, Linton and Main (1997) have suggested a cutoff score of 105, above which acute low back pain patients should be considered to be at significantly greater risk for the development of chronic low back pain.

$\underline{\text { Blue Flags }}$ 
Main (2002) has been very influential in increasing awareness that certain working conditions and adverse workplace characteristics may place an individual at increased risk of disability and associated absences from work. Such features of work that are perceived to be aversive in nature may present a major obstacle to recovery, and will ultimately delay improvement in functioning and return-to-work. For example, if disgruntled workers injure their backs, they are more likely to take an extended leave of absence from work, relative to workers who are more satisfied with their place of employment. As noted earlier, any prolonged leave of absence from work may be especially problematic because the longer an individual is out of work, the less likely he or she is to return. Indeed, as Bigos and colleagues (1990) have noted, once an individual is off work, then perception about symptoms, safety of return-to-work, and the impact of return-to-work on one's personal life and safety can all affect recovery even in the "most well-meaning worker."

The delineation of blue flags, or the person's perceptions regarding the work environment, has been largely based upon previous literature pertaining to stress and control. These blue flags refer to perceived occupational factors that are believed to impede an individual's recovery. The basic blue flags delineated by Main include the following:

- A high demand/low control work environment in which workers perceive they are in a stringent, inflexible environment where they have little control over what is going on but, at the same time, are expected to be highly productive.

- The perception that the style of management is unhelpful.

- The belief that they working under a perceived time pressure.

- The belief that they receive poor social support from their colleagues.

- They dislike their jobs. 
Because these above blue flags are relatively new, there are no standard guidelines that have yet been developed to assess them, although initial attempts are being made (e.g., Jocksimovic, Starke, Knesebeck, \& Siegrist, 2002). However, it should be kept in mind that because work plays a significant role in individuals' lives, a great many interdisciplinary pain management programs have begun to include work-related components in their overall treatment strategy.

\section{Black Flags}

In contrast to the above blue flags, which are mostly subjective in nature, black flags refer to more objective occupational factors that affect all workers equally. It may, though, sometimes be difficult to differentiate between subjective and objective occupational stress factors, because they may work independently or in combination with one another. Nevertheless, black flags include both nationally established policies concerning the conditions of employment and sickness policy, as well as working conditions that are specific to a particular organization. Thus, when one is assessing these objective occupational risk factors, careful attention should be given to both the national and local level policies. Even though methods for their assessment have not been operationalized in standard guidelines, a number of black flags have been identified. On the national level, these include the following: salary rates such as the minimum wage; nationally negotiated entitlements related to sick certification; benefit systems; and wage reimbursement rates. On the local level, the company's sickness policy includes the following: workers' entitlement to sick leave; the role of occupational health and "signing off" and "signing on" requirements for full fitness; the possibility and availability of work accommodations. 
In addition to the above, there are also a number of content-specific features of work that appear to serve as black flags. These include the following:

- Temporal characteristics (such as number of working hours and shift patterns) should be evaluated.

- Variables pertaining to ergonomics (such as job weight lifting levels, lifting frequency, sustained positions and maintaining postures, and required sitting or standing) are all viewed as potentially serving as contributors to the development of chronicity.

These above content-specific occupational risk factors appear to be far more important for evaluating disability risks than for predicting the onset of pain (Anderrson, Pope, Frymoyer, \& Snook, 1991). That is to say, although these types of factors may not have initially led to the onset of pain, they appear to promote disability once the pain has occurred. Therefore, in order to increase the probability of injured workers remaining on the job, employers should make reasonable accommodations in order to reduce the level of low back pain discomfort that their injured workers face (Shaw, Pransky, Fitzgerald, Patterson, \& Winters, 2002).

\section{THE GOAL OF EARLY INTERVENTION}

There is now a growing agreement among clinical researchers that the most effective method to prevent the development of chronic musculoskeletal disorders is to provide appropriate primary and secondary intervention. Gatchel and colleagues have addressed this important issue. The main goal of an initial study (1995) was to identify predictors of when acute low back pain occurrences were likely to develop into chronic disability problems. The results of that initial project clearly isolated some significant psychosocial risk factors that successfully predicted the development of chronicity during a one-year follow-up. Using a receiver-operator characteristic (ROC) curve analysis, which was based on the probabilities 
estimated from the logistic regression model developed on the large cohort of patients evaluated during that project, a statistical algorithm was developed that could be used to identify (with a 90.7\% accuracy rate) "high risk" patients who were prime candidates for early intervention in order to prevent chronicity. As an extension of these important findings, a second study (Gatchel et al., 2003) involved the assessment of a large cohort of acute low back pain patients in order to screen out those patients who were at high risk for developing chronicity (using the aforementioned algorithm). These high-risk patients were then randomly assigned to one of two groups: an early intervention group or a non-intervention group. During the next year, routine three-month follow-up evaluations were conducted in order to assess important long-term socioeconomic outcomes, such as return-to-work, health care utilization rates, medication use rates, etc. It was hypothesized that early intervention at the acute stage would prevent the development of chronic disability. It should also be noted that, as a replication of the previous grant project results, the non-intervention group was compared to a demographically-matched cohort of initially assessed patients who did not display the "high risk" profile. It was hypothesized that the high-risk non-intervention group patients would demonstrate higher rates of chronic disability at one year relative to the low or "not-at-risk" profile patients.

Another study emanating from this project further characterized those patients who were classified as high risk or low risk, based upon our classification algorithm (Pulliam, Gatchel, \& Gardea, 2001). Results clearly revealed additional mental health differences between these two groups. The high-risk patients were found to have lower scores on positive temperament (i.e., less energy, enthusiasm and optimism when undertaking projects) as measured on the Schedule for Nonadaptive and Adaptive Personality (Clark, 1993), greater reliance on an avoidance coping strategy as assessed on the Ways of Coping Questionnaire-Revised (Vitaliano, Russo, Carr, 
Maiuro, \& Becker, 1985), and a greater prevalence of a DSM-IV Axis I Disorder. These findings, therefore, again highlight the fact that the identified high-risk patients have a stronger potential for psychosocial factors that may contribute to chronic mental and physical health disability if not managed in a timely fashion. Moreover, a just-completed analysis of a larger cohort of patients from this project (and not just those who participated in the early intervention component of the study) has indicated that the high-risk subjects had a higher prevalence of Mood Disorders, Anxiety Disorders, Somatoform/Pain Disorders, Substance Abuse/Dependence Disorders, and Co-morbid Axis I and Axis II Disorders.

The early intervention approach evaluated in this above study was based upon a number of suggestions by leading experts in the field that, in order to decrease the high cost of chronic musculoskeletal disability, there is a great need for better identifying patients at the acute phase who would benefit from such early intervention (Hazard, 1995; Linton, 2002; Linton \& Bradley, 1996). Moreover, the early detection and intervention in order to prevent chronic disorders has also been recognized as a high priority research area (Human Capital Initiative Coordinating Committee, 1996). Also, as noted earlier, with the realization that work-related musculoskeletal disorders was a major socioeconomic problem in this country, a series of questions posed by the U.S. Congress prompted the National Institutes of Health to request the National Research Council to examine ways of reducing this problem of work-related musculoskeletal disorders. This resulted in a publication, which, as one of its conclusions, highlighted the importance of early intervention in order to reduce the development of chronic disability (National Research Council, 2001).

The results of our study clearly indicated that early intervention at the acute stage of low back pain significantly reduced the prevalence of chronic disability, relative to those high-risk 
patients who did not receive such early intervention (Gatchel et al., 2003). Table 3 summarizes the outcome data that displayed significant differences among groups in the anticipated directions. As can be seen, the high-risk patients who received early intervention (the HR-I group) displayed significantly fewer indices of chronic pain disability on a wide range of work, health care utilization, medication use and self-reported pain variables, relative to the high-risk patients who did not receive such early intervention (the HR-NI group). Relative to the HR-NI group, the HR-I group was much more likely to have returned to work (odds ratio=4.55), less likely to be currently taking narcotic analgesics (odds ratio=0.44), and also less likely to be taking psychotropic medication (odds ratio=0.24). In addition, the HR-NI group also displayed significantly more symptoms of chronic pain disability on these variables relative to the initially low-risk low back pain patients (the LR group).

\section{INSERT TABLE 3 ABOUT HERE}

The cost-comparison savings data from this study were also quite impressive. Using unit cost multipliers obtained from the Bureau of Labor Statistics for compensation costs due to disability days (Bureau of Labor Statistics, 2002), from the Medical Fees in the United States 2002 (Medical Fees in the United States 2000, 2002) for health care costs, and the Drug Topics

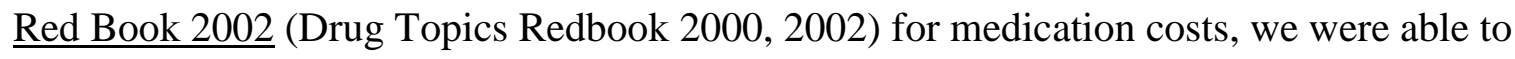
calculate the average costs per patient associated with health care visits related to low back pain, narcotic analgesic and psychotropic medications, and work disability days/lost wages. Table 4 lists these costs associated with the HR-I and HR-NI groups. As can be seen, the average overall 
costs per patient over the one-year follow-up period (even taking into account the $\$ 3,885 /$ patient cost of the early intervention for the HR-I group) was significantly higher for the HR-NI group.

\section{INSERT TABLE 4 ABOUT HERE}

\section{SUMMARY AND CONCLUSIONS}

The biopsychosocial approach to musculoskeletal pain and disability has been reviewed, emphasizing the complex and dynamic interaction among physiologic, psychologic and social factors that perpetuate and may even worsen the clinical presentation. This complexity is highlighted by the "red, yellow, blue and black flags" that have been developed for assessing acute low back pain patients who have potentially significant risk factors for developing chronic disability problems. The importance of taking into account such potential risk factors in primary and secondary interventions was emphasized. Finally, an example of a series of studies using an early risk identification and intervention program was presented. Such programs show great promise for the most treatment- and cost-effective approach for musculoskeletal disorders such as low back pain. 


\section{REFERENCES}

ACC and the National Health Committee. (1997). New Zealand Acute Low Back Guide. Wellington, New Zealand.

Anderrson, G. B. J., Pope, M. H., Frymoyer, J. W., \& Snook, S. H. (1991). Occupational Low Back Pain: Assessment, Treatment, and Prevention. In M. H. Pope, G. B. J. Anderrson, J. W. Frymoyer \& D. B. Chaffin (Eds.), Epidemiology and Cost. St. Louis: Mosby Year Book.

Bigos, S. J., Battie, M. C., Nordin, M., Spengler, D. M., \& Guy, D. P. (1990). Industrial Low Back Pain. In J. Weinstein \& S. Wiesel (Eds.), The Lumbar Spine (pp. 846-859). Philadelphia: W.B. Saunders and Co.

Bureau of Labor Statistics. (2002). 2002, from http://data.bls.gov/surveymost

Clark, L. (1993). Schedule for Nonadaptive and Adaptive Personality (SNAP). Minneapolis: University of Minneapolis Press.

Drug Topics Redbook 2000. (2002). Montvale, NJ: Thompson Medical Economics.

Gatchel, R. J., Polatin, P. B., \& Mayer, T. G. (1995). The dominant role of psychosocial risk factors in the development of chronic low back pain disability. Spine, 20(24), 2702-2709.

Gatchel, R. J., Polatin, P. B., Noe, C. E., Gardea, M. A., Pulliam, C., \& Thompson, J. (2003). Treatment- and cost-effectiveness of early intervention for acute low back pain patients: A one-year prospective study. Journal of Occupational Rehabilitation, 13, 1-9.

Hazard, R. G. (1995). Spine update: Functional restoration. Spine, 20, 2345-2348.

Human Capital Initiative Coordinating Committee. (1996). Doing the Right Thing: A Research Plan for Healthy Living. Washington: American Psychological Association. 
Jocksimovic, L., Starke, D., Knesebeck, O., \& Siegrist, J. (2002). Perceived work stress, overcommitment, and self-reported musculoskeletal pain: A cross-sectional investigation. International Journal of Behavioral Medicine, 9, 122-138.

Kendall, N. A. S., Linton, S. J., \& Main, C. J. (1997). Guide to assessing psychosocial yellow flags in acute low back pain: risk factors for long-term disability and work loss. In Accident Rehabilitation and Compensation Insurance Corporation of New Zealand and the National Health Committee.

Linton, S. J. (2002). A cognitive-behavioral approach to the prevention of chronic back pain. In D. C. Turk \& R. J. Gatchel (Eds.), Psychological Approaches to Pain Management: A Practitioner's Handbook (2nd ed.). New York: Guilford.

Linton, S. J., \& Bradley, L. A. (1996). Strategies for the prevention of chronic pain. In R. J. Gatchel \& D. C. Turk (Eds.), Psychological Approaches to Pain Management: A Practitioner's Handbook. New York: Guilford Publications, Inc.

Main, C. J. (2002). Concepts of Treatment and Prevention in Musculoskeletal Disorders. In S. J. Linton (Ed.), New Avenues For The Prevention Of Chronic Musculoskeletal Pain And Disability. Amsterdam: Elsevier Science B.V.

Mayer, T. G., \& Gatchel, R. J. (1988). Functional Restoration for Spinal Disorders: The Sports Medicine Approach. Philadelphia: Lea \& Febiger.

Mayer, T. G., Gatchel, R. J., Mayer, H., Kishino, N. D., Keeley, J., \& Mooney, V. A. (1987). A prospective two-year study of functional restoration in industrial low back injury. An objective assessment procedure [published erratum appears in JAMA 1988 Jan 8;259(2):220]. JAMA, 258(13), 1763-1767. 
Mayer, T. G., Gatchel, R. J., \& Polatin, P. B. (Eds.). (2000). Occupational Musculoskeletal Disorders: Function, Outcomes and Evidence. Philadelphia: Lippincott Williams \& Wilkins.

Mayer, T. G., Polatin, P., Smith, B., \& et al. (1995). Contemporary concepts in spine care rehabilitation: Secondary and tertiary nonoperative care. Spine, 20, 2060-2066.

McGuirk, B., King, W., Govind, J., Lowry, J., \& Bogduk, N. (2001). Safety, efficacy and costeffectiveness of evidence-based guidelines for the management of acute low back pain in primary care. Spine, 26, 2615-2622.

Medical Fees in the United States 2000. (2002). Los Angeles: Practice Management Information Corporation.

National Research Council. (2001). Musculoskeletal Disorders and the Workplace: Low Back and Upper Extremities. Washington, D.C.: National Academy Press.

Pulliam, C., Gatchel, R. J., \& Gardea, M. A. (2001). Psychosocial differences in high risk versus low risk acute low back pain differences. Journal of Occupational Rehabilitation, 11, 4352.

Shaw, W. S., Pransky, G. S., Fitzgerald, T. E., Patterson, W., \& Winters, T. (2002). Returning to work after acute occupational low back pain: A multiple site study of prognosis.

Montreal: Montreal International Forum V for Primary Care Research on Low Back Pain.

Turk, D. C., \& Gatchel, R. J. (Eds.). (2002). Psychological Approaches to Pain Management: A Practitioner's Handbook (2nd ed.). New York: Guilford.

Turk, D. C., \& Monarch, E. S. (2002). Biopsychosocial perspective on chronic pain. In D. C. Turk \& R. J. Gatchel (Eds.), Psychological Approaches to Pain Management: A Practitioner's Handbook (2nd ed.). New York: Guilford. 
Vitaliano, P., Russo, J., Carr, J., Maiuro, R., \& Becker, J. (1985). The ways of coping checklist: Revision and psychometric properties. Multivariate Behavioral Research, 20, 3-26. 
Table 1. Summary of the Risk Factors or "Flag" Categories

RED FLAGS Potentially significant physiological risk factors for developing chronic low back pain if not appropriately assessed

YELLOW FLAGS Potentially significant psychosocial risk factors for developing chronic low back pain

BLUE FLAGS Perceived occupational factors believed by patients to impede their recovery

BLACK FLAGS Objective occupational workplace factors that may initially lead to the onset of low back pain, and may promote disability once the acute episode has occurred. 
Table 2. Important Components in Primary Intervention for Acute Low Back Pain

- In order to gain symptomatic relief of pain, simple analgesics and NSAIDs should be prescribed.

- Instead of taking medication on an "as needed" basis, patients should be told to take medication at regular intervals because this type of administration has been found to effectively reduce the symptoms associated with simple backache. Narcotics should be avoided altogether whenever possible because of their potential adverse side effects and potentially addictive qualities. If narcotics are needed, their use should not exceed a period of more than two weeks.

- Physical therapy may be used to help alleviate the pain. Aerobic, endurance training exercises (such as cycling, walking, swimming and even light jogging) are especially helpful in maintaining a patient's muscular integrity; and they also serve to prevent the occurrence of physical deconditioning. These exercises are usually easily tolerated by most patients with simple back pain, and may begin within the first two weeks of pain onset. Exercises designed to strengthen the abdominal and back muscles may be added gradually, but begin no earlier than two weeks after pain onset.

- Manipulation conducted within the first six weeks of pain onset may provide short term improvement in pain, and may ultimately encourage patients to encourage in daily activities or exercise that might be avoided if the pain were not first reduced.

- Patients should be encouraged to remain physically active. At this time, bed rest for simple back pain is typically contraindicated, because prolonged bed rest has been 
deemed to be potentially harmful and, may in fact, contribute to the development of chronic pain disability. Under certain instances, bed rest may be warranted; however, because of the potential negative effects, such bed rest should not exceed the recommended duration of 2-3 days.

- Patients should be advised to continue carrying out their normal activities, in spite of the pain they may experience.

- The primary care physician should assure patient that, even though they may likely experience some pain in response to their normal activities, the pain is only temporary and is not indicative of harm or damage. 
Table 3. Long-Term Outcome Results at 12-Month Follow-up (from Gatchel et al., 2003)

OUTCOME MEASURE

$\%$ Return-to-Work at Follow-up*

Average \# Health Care Visits Regardless of Reason**

Average \# Health Care Visits Related to LBP**

Average \# of Disability Days Due to Back Pain**

Average of Self-Rated Most “Intense Pain” at 12-

Month Follow-Up (0-100 scale)**

Average of Self-Rated Pain Over Last 3 Months (0-

$$
100 \text { scale) })^{* *}
$$

\% Currently Taking Narcotic Analgesics*

\% Currently Taking Psychotropic Medication*

$\begin{array}{cccc}\text { HR-I } & \text { HR-NI } & \text { LR } & \\ \underline{(\mathbf{n = 2 2})} & \underline{(\mathbf{n = 4 8})} & \underline{(\mathbf{n}=\mathbf{5 4})} & \mathbf{p} \text { Value } \\ 91 \% & 69 \% & 87 \% & .027 \\ 25.6 & 28.8 & 12.4 & .004 \\ 17.0 & 27.3 & 9.3 & .004 \\ 38.2 & 102.4 & 20.8 & .001 \\ 46.4 & 67.3 & 44.8 & .001 \\ & & & \\ 26.8 & 43.1 & 25.7 & .001 \\ & & & \\ 27.3 \% & 43.8 \% & 18.5 \% & .020 \\ 4.5 \% & 16.7 \% & 1.9 \% & .019\end{array}$

* Chi-square analysis ** ANOVA 
Table 4. Cost-Comparison Results (Average Cost Per Patient/YEAR; from Gatchel et al., 2003)

\section{COST VARIABLE}

Health care Visits Related to LBP

Narcotic Analgesic Medication

Psychotropic Medication

Work Disability Days/Lost Wages

Early Intervention Program
HR-I

$\underline{(\mathrm{n}=\mathbf{2 2})}$

$\$ 1,670$

$\$ 70$

$\$ 24$

$\$ 7,072$

$\$ 3,885$
HR-NI

$\underline{(n=48)}$

$\$ 160$

$\$ 55$

$\$ 18,951$

NA

TOTALS

$\$ 12,721$

$\$ 21,843$ 